\title{
Mathematical Modeling of Control Systems Based on Output Feedback Decoupling, Curve Fitting and Spline Interpolation
}

\author{
Chi Zhang ${ }^{1, a}$, Hong Wang ${ }^{2, b}$, Xiaofei Lian ${ }^{3, c}$ \\ $1,2,3$ Northeastern University, 110004 Shenyang, China \\ àhch_angi@163.com, bhongwang@mail.neu.edu.cn
}

Keywords: Mathematical modeling, output feedback decoupling, curve fitting, spline interpolation

Abstract. In this paper, based on the output feedback decoupling, curve fitting and spline interpolation method, a mathematical model is presented for nonlinear dynamical system wherein only system inputs and outputs are known.

\section{Intorduction}

A proper mathematical model of the system we want to control is essential to many applications such as system analysis, controller design and optimization [1,2]. However, Control systems with nonlinearity and dynamicity are usually difficult to find out their mathematical models. This calls for a theory on the solution of mathematical modeling problem by new methods.

The system is said to be input-output decoupled if each of its inputs influences one and only one of its outputs-to-be-controlled [3]. The problems of dynamical modelling of large-scale systems are considered with a view to developing reduced-order models using aggregation and decoupling [4]. Curve fitting has been studied extensively and a number of approaches have been employed [5]. In the usual setting, some data points are given and one wants to find a function with the minimum violations measured by a norm in the given data points. In these approaches, norms are applied to aggregate all violations as a scalar (see for example [6,7]). The theory of B-splines is a well developed area of applied numerical analysis and interpolation theory, and the use of B-splines rivals that of Bezier curves in applicability to computer graphics and approximation theory [8].

These approaches can be used to build the model. The objective of this paper is to describe the methodology and demonstrate its effectiveness using an example problem. The remainder of this paper is organized as follows: the general formulation is described in Section 1, the new method is used to solve a control system modeling problem in Section 2, and conclusions are given in Section 3.

\section{Mathematical modeling}

\subsection{Final value theorem refinemen}

\section{Theorem 1.1}

In mathematical analysis, the final value theorem (FVT) is one of several similar theorems used to relate frequency domain expressions to the time domain behavior as time approaches infinity. A final value theorem allows the time domain behavior to be directly calculated by taking a limit of a frequency domain expression, as opposed to converting to a time domain expression and taking its limit $[9,10,11]$.

Mathematically, if

$$
\lim _{t \rightarrow \infty} f(t)
$$

exists, then

$$
\lim _{t \rightarrow \infty} f(t)=\lim _{s \rightarrow 0} s F(s),
$$

where $F(\mathrm{~s})$ is the laplace transform of the signal $f(t)$. 


\section{Deduction 1.2}

The final value of output is equivalent to the final value of input multiplied by the ratio of the numerator constant and the denominator constant of the transfer function.

Mathematically, if

$$
\mathrm{G}(s)=\frac{a_{n} s^{n}+a_{n-1} s^{n-1}+\cdots+a_{1} s+a_{0}}{s^{n}+b_{n-1} s^{n-1}+\cdots+b_{1} s+b_{0}}
$$

exists, then

$$
\lim _{t \rightarrow \infty} y(t)=\frac{a_{0}}{b_{0}} \cdot \lim _{t \rightarrow \infty} u(t) .
$$

Proof.

Consider the application of theorem 2.1,

$$
\begin{aligned}
\lim _{t \rightarrow \infty} y(t) & =\lim _{s \rightarrow 0} s Y(s) \\
& =\lim _{s \rightarrow 0} s \cdot U(s) G(s) \\
& =\lim _{s \rightarrow 0} s U(s) \cdot \lim _{s \rightarrow 0} G(s) \\
& =\lim _{t \rightarrow \infty} u(t) \cdot \lim _{s \rightarrow 0} G(s) \\
& =\frac{a_{0}}{b_{0}} \lim _{t \rightarrow \infty} u(t) .
\end{aligned}
$$

\subsection{Formulation}

We consider a nonlinear control system $\Sigma$ of the form

$$
\Sigma\left\{\begin{array}{l}
\dot{x}=f(x)+g(x) u, \\
y=h(x)
\end{array}\right.
$$

where $\mathrm{x}=\operatorname{col}(\mathrm{x} 1, \ldots, \mathrm{xn}) \in \mathrm{R}^{\mathrm{n}}$ are local coordinates for the state space manifold $\mathrm{M}, \mathrm{u} \in \mathbf{R}^{\mathrm{m}}$ denotes the controls, $y \in \mathbf{R}^{\mathrm{m}}$ denotes the outputs-to-be-controlled. We will assume throughout that the vector fields $\mathrm{f}, \mathrm{g}_{1}, \ldots, \mathrm{g}_{\mathrm{m}}$ and the mappings $\mathrm{h}: \mathrm{M} \rightarrow \mathbf{R}^{\mathrm{m}}, \mathrm{k}: \mathrm{M} \rightarrow \mathbf{R}^{\mathrm{q}}$ are meromorphic [3].

The transfer function matrix is

$$
\begin{aligned}
G(s) & =\left[\begin{array}{cccc}
G_{11}(s) & G_{12}(s) & \cdots & G_{1 r}(s) \\
G_{21}(s) & G_{22}(s) & \cdots & G_{2 r}(s) \\
\vdots & \vdots & & \vdots \\
G_{m 1}(s) & G_{m 2}(s) & \cdots & G_{m r}(s)
\end{array}\right] . \\
G_{i j}(s) & =\frac{a_{i j, n} s^{n}+a_{i j, n-1} s^{n-1}+\cdots+a_{i j, 1} s+a_{i j, 0}}{s^{n}+b_{i j, n-1} s^{n-1}+\cdots+b_{i j, 1} s+b_{i j, 0}}
\end{aligned}
$$

stands for the $j$-th input's influence on the i-th output, where $i=1,2, \cdots, m, j=1,2, \cdots, r$.

According to Eq. 4, we have

$$
\frac{a_{i j, 0}}{b_{i j, 0}}=\frac{\lim _{t \rightarrow \infty} y_{i}(t)}{\lim _{t \rightarrow \infty} u_{j}(t)} \text {. }
$$


For given inputs, we can find each parameter's influence on output curves within a certain range. Then $a_{i j, n}, a_{i j, n-1}, \cdots, a_{i j, 1}, b_{i j, n-1}, b_{i j, n-2}, \cdots, b_{i j, 1}$ will be determined by spline interpolation and curve fitting.

Firstly, we provide the initial value of parameters. With the $\beta$ spline interpolation, the controled variables are obtained at the moment of a new time series $t i=0$ : time samples: length $(t)$. Time samples must be evenly spaced. Then work out the system output response for the obtained inputs and draw the response curve. Finally, adjust the response curve by the parameters in order to bring the errors between the response curve and the actual output curve within prescribed limits. If the system is the PID control system, the transfer function of the PID controller is

$$
G_{P I D}=K_{p}+\frac{K_{i}}{s}+K_{d} s
$$

as the structure of control system is known, the control object transfer function $\mathrm{G}_{0}$ can be obtained.

\section{Example}

We illustrate the theory developed in the previous section by means of an example. Consider the following system:

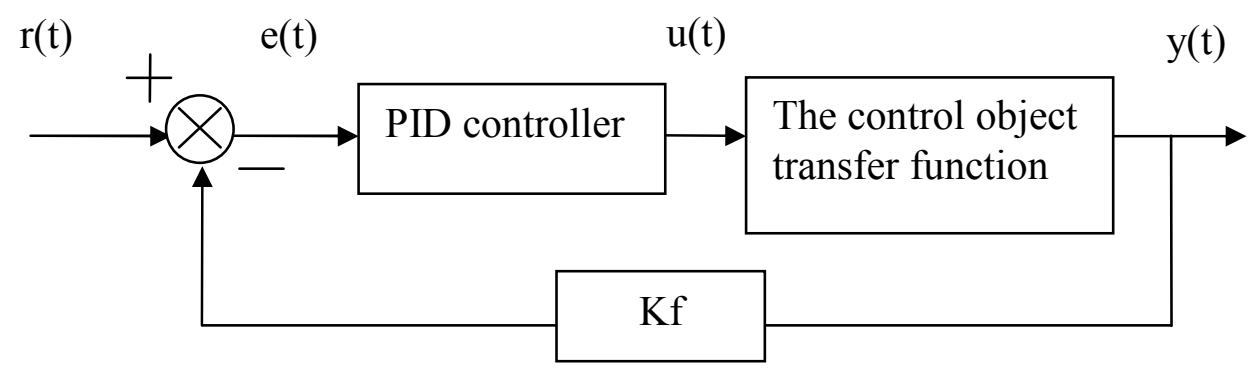

Fig. 1. The control system frame

The data of system input and output can be gotten through experiments.

The transfer function of the PID controller is

$$
G_{P I D}=K_{p}+\frac{K_{i}}{S}+K_{d} S \text {. }
$$

The control object transfer function is

$$
G_{0}=\frac{K}{S^{2}+a S+b},
$$

where K, a, b, Kf are unknown parameters. We obtain the transfer function of the system:

$$
G=\frac{G_{0} G_{P I D}}{1+K f \cdot G_{0} G_{P I D}}=\frac{K_{d} K S^{2}+K_{p} K S+K_{i} K}{S^{3}+\left(a+K f \cdot K_{d} K\right) S^{2}+\left(b+K f \cdot K_{p} K\right) S+K f \cdot K_{i} K} .
$$

We then assume

$$
G=\frac{a_{2} S^{2}+a_{1} S+a_{0}}{S^{3}+b_{2} S^{2}+b_{1} S+b_{0}} .
$$


State space expression:

$$
\begin{aligned}
& {\left[\begin{array}{c}
\mathrm{x}_{1} \\
\mathrm{x}_{2} \\
\mathrm{x}_{3}
\end{array}\right]=\left[\begin{array}{rrr}
0 & 1 & 0 \\
0 & 0 & 1 \\
-b_{0} & -b_{1} & -b_{2}
\end{array}\right]\left[\begin{array}{l}
c_{1} \\
c_{2} \\
c_{3}
\end{array}\right] u} \\
& y=\left[\begin{array}{lll}
1 & 0 & 0
\end{array}\right]\left[\begin{array}{l}
x_{1} \\
x_{2} \\
x_{3}
\end{array}\right] .
\end{aligned}
$$

We then obtain

$$
y(t)=C \cdot\left(e^{A t} x_{0}+\int_{0}^{t} e^{A(t-\tau)} \cdot B \cdot u \cdot d \tau\right)
$$

where

$$
A=\left[\begin{array}{ccc}
0 & 1 & 0 \\
0 & 0 & 1 \\
-K f \cdot K_{i} K & -\left(b+K f \cdot K_{p} K\right) & -\left(a+K f \cdot K_{d} K\right)
\end{array}\right]
$$

is system matrix,

$$
B=\left[\begin{array}{c}
K_{p} K_{d} K \\
K_{p} K-K_{p} K_{d} K\left(a+K f \cdot K_{p} K_{d} K\right) \\
K_{i} K-K_{p} K\left(a+K f \cdot K_{p} K_{d} K\right)+K_{p} K_{d} K\left(a+K f \cdot K_{p} K_{d} K\right)^{2}-K_{p} K_{d} K\left(b+K f \cdot K_{p} K\right)
\end{array}\right]
$$

is input matrix,

$$
C=\left[\begin{array}{lll}
1 & 0 & 0
\end{array}\right]
$$

is output matrix,

$$
\mathrm{D}=0
$$

is direct transfer matrix.

As mentioned in the last paragraph of the previous, with the $\beta$ spline interpolation, the control variable is obtained at the moment of a new time series $t_{1}=0$ : time samples: length $(t)$.

In the Eq. 14, $\mathrm{a}_{2}=\mathrm{Ki} \cdot \mathrm{K}, \mathrm{b}_{2}=\mathrm{Kf} \cdot \mathrm{Ki} \cdot \mathrm{K}$. Using Eq. 4, we have

$$
\frac{\lim _{t \rightarrow \infty} u(t)}{\lim _{t \rightarrow \infty} y(t)}=\frac{b_{2}}{a_{2}}=K f .
$$

$\mathrm{K}$, $\mathrm{a}, \mathrm{b}$ can be obtained by repeatedly adjusting. a is proportional to the overshoot and the peak time. $\mathrm{b}$ is inversely proportional to the peak. $\mathrm{K}$ is inversely proportional to the overshoot. The response curve is also adjusted by the parameters in order to bring the errors between the response curve and the actual output curve within prescribed limits.

When $\lim _{t \rightarrow \infty} y(t)=1$, we carried out experiments to get $\lim _{t \rightarrow \infty} u(t)=62.76$. In the Eq. $13, \mathrm{Kf}=62.76$. Further, we obtain $\mathrm{K}=0.38, \mathrm{a}=1, \mathrm{~b}=0.1$. When $\lim _{t \rightarrow \infty} y(t)=0.8$, we carried out experiments to get $\lim _{t \rightarrow \infty} u(t)=57.44$. In the Eq. $13, \mathrm{Kf}=71.8$. Further, we obtain $\mathrm{K}=0.38, \mathrm{a}=1, \mathrm{~b}=0.1$. Thus, the feasibility of the model is validated. 

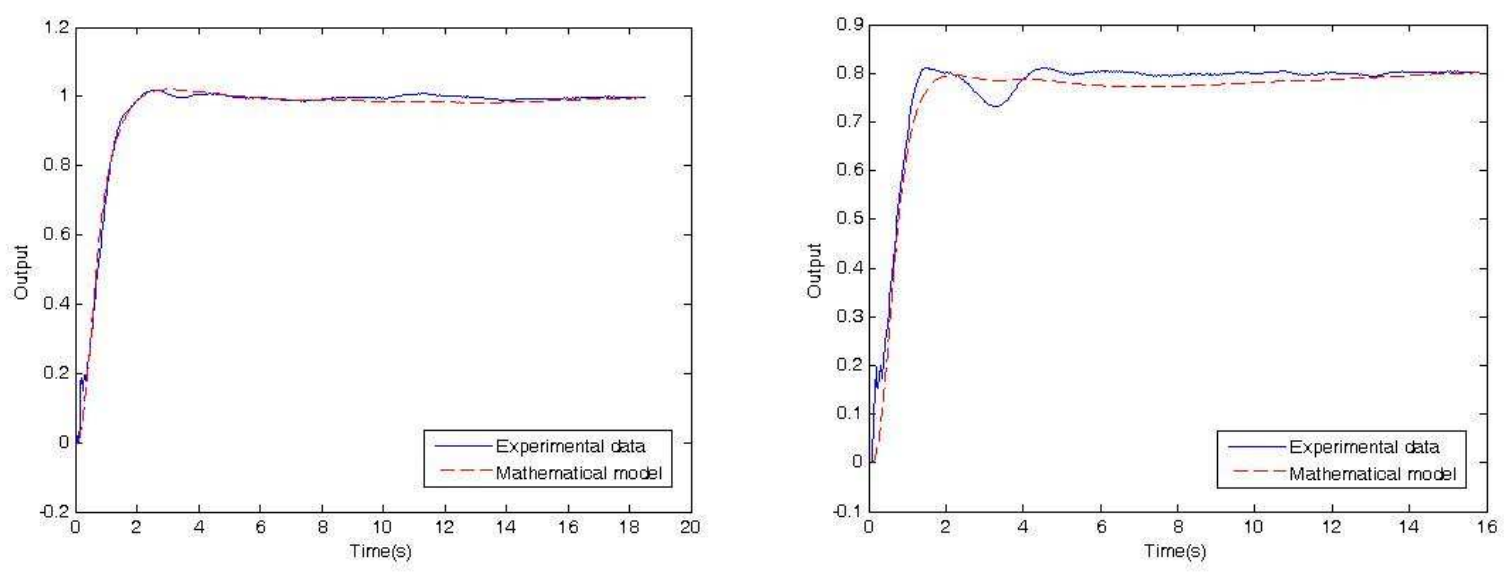

Fig. 2. Output fitting curve

The dashed line is the output response curve which is drawn out by analyzing the massive data gained from the model (Fig.2). The calculated results are compared with different experimental data, which show that the model raised is reliable. Furthermore, implemented in the optimization by modeling, the system performs better.

\section{Summary and conclusions}

A new method for mathematical modeling of control systems with nonlinearity and dynamicity has been developed. The rationality and the accuracy of the method are verified through the real experiments.

\section{Acknowledgment}

This work was supported by National Science Foundation of China (61071057), Fundamental Research Funds for the Central Universities of China (N100603003).

\section{References}

[1] K. Schlacher, Mathematical modeling for nonlinear control: a Hamiltonian approach, Mathematics and Computers in Simulation 79 (2008) 829-849.

[2] Han-Xiong Li, Chenkun Qi, Modeling of distributed parameter systems for applications-A synthesized review from time-space separation, Journal of Process Control 20 (2010) 891-901.

[3] H.J.C. Huijberts, C.H. Moog, R. Pothin, Input-output decoupling of nonlinear systems by static measurement feedback, Systems \& Control Letters 39 (2000) 109-114.

[4] T. S. Dillon, Dynamical modelling and control of large-scale systems, International Journal of Electrical Power \& Energy Systems 4 (1982) 29-36.

[5] L. Pourkarimi, M.A. Yaghoobi, M. Mashinchi, Efficient curve fitting: An application of multiobjective programming, Applied Mathematical Modelling 35 (2011) 346-365.

[6] J. Stoer, R. Bulirsch, Introduction to Numerical Analysis, second ed, Springer-Verlag, Berlin, 1972.

[7] E. Sli, D.F. Mayers, An Introduction to Numerical Analysis, Cambridge University Press, 2003.

[8] Hiroyuki Kano, Magnus Egerstedt, Hiroaki Nakata, Clyde F. Martin, B-splines and control theory, Applied Mathematics and Computation 145 (2003) 263-288.

[9] Franklin G. F., Emami-Naeini A., and Powell J. D., Feedback Control of Dynamic Systems, third ed, Addison-Wesley Longman Publishing Co., 1993.

[10] Joel L. Schiff, The Laplace transform: theory and applications, Springer-Verlag, New York, 1999

[11] S. B. Karmakar, Approximate analysis of non-linear systems by Laplace transform, Journal of Sound and Vibration 69 (1980) 597-602. 